\title{
GLICERÓLISE ENZIMÁTICA DE ÓLEOS DE SOJA ASSISTIDA POR ULTRASSOM EM MEIO LIVRE DE SOLVENTE ORGÂNICO SOB AGITAÇÃO
}

\author{
D. REMONATTO ${ }^{1}$,L. A. LERIN ${ }^{1}$, K. A. ONISHI ${ }^{1}$, V. OENNING NETO ${ }^{1}$, D. OLIVEIRA ${ }^{1}$, \\ J. L. NINOW ${ }^{1}$ \\ ${ }^{1}$ Universidade Federal de Santa Catarina, \\ Departamento de Engenharia Química e Engenharia de Alimentos \\ E-mail para contato: daniremonatto@gmail.com
}

\begin{abstract}
RESUMO - Este trabalho apresenta dados experimentais da glicerólise de óleo de soja utilizando como catalisador a enzima Novozym 435 em sistema livre de solvente e em ultrassom, para a produção de emulsificantes, monoacilgliceróis (MAGs) e diacilgliceróis (DAGs). Os experimentos foram realizados em modo batelada visando o estudo do efeito das variáveis de processo: temperatura $\left(40\right.$ a $\left.70{ }^{\circ} \mathrm{C}\right)$, concentração de enzima $(2,5$ a $10 \%$, $\mathrm{m} / \mathrm{m}$, em relação aos substratos), razão molar de glicerol: óleo $(0,8: 1$ a 3:1), agitação (0 a $1200 \mathrm{rpm}$ ) e potência do ultrassom (0 a 100\%). Óleo de soja teve seus melhores resultados de conteúdo de MAGs + DAGs $(\sim 65 \% \mathrm{~m} / \mathrm{m})$ quando se utilizou a relação molar de glicerol:óleo de 0,8:1, $70{ }^{\circ} \mathrm{C}, 600 \mathrm{rpm}, 90$ minutos de reação, concentração de enzima de $10 \%(\mathrm{~m} / \mathrm{m})$ e $40 \%$ de potência de ultrassom. Este estudo apontou a glicerólise catalisada por lipase em meio livre de solvente com auxílio do ultrassom pode ser uma potencial rota para a produção de altos conteúdos de MAGs e DAGs.
\end{abstract}

\section{INTRODUÇÃO}

A busca por processos alternativos para a síntese de produtos de interesse para a indústria de alimentos, farmacêutica e cosmética, que não causem ou reduzam os danos ao ambiente, vêm despertando grande interesse de pesquisadores em todo o mundo. Uma alternativa é a síntese enzimática, que apresenta muitas vantagens em relação à síntese química (Lerin, 2010). Diversas pesquisas têm sido focadas na modificação de óleos e gorduras para a obtenção de produtos que aliem propriedades funcionais e/ou de saúde, bem como propriedades tecnológicas que permitam sua utilização nas linhas de processamento (Xu, 2004; Feltes et al., 2009). Entre as técnicas existentes, a glicerólise enzimática, para a produção de monoacilgliceróis (MAGs) e diacilgliceróis (DAGs), tem sido alvo de muitos estudos, pois estes glicerídeos são excelentes emulsificantes e moléculas condicionadoras utilizadas na indústria.

Porém a reação de glicerólise apresenta um inconveniente, a imiscibilidade dos substratos, que prejudica os rendimentos da reação. Os efeitos físicos do ultrassom, como o aumento da temperatura, a transferência de massa, a cavitação estável e a transitória vêm a contribuir para o aumento da miscibilidade das fases óleo e glicerol, assim é possível reduzir o tempo de reação e maximizar a produção de monoacilgliceróis e diacilgliceróis. A influência das ondas ultrassônicas na atividade e 


\section{9 a 22 de outubro de 2014 \\ Florianópolis/SC}

estabilidade de enzimas tem demonstrado ser específica para cada enzima e dependente dos parâmetros de sonicação (Martinez et al., 2000).

Neste contexto, o presente estudo teve como objetivo geral avaliar a potencialidade da produção enzimática de emulsificantes de grau alimentício, monoacilgliceróis e diacilgliceróis, através da glicerólise de óleo de soja, e glicerol, em meio livre de solvente em sistema de ultrassom, utilizando uma lipase comercial imobilizada, Novozym ${ }^{\circledR} 435$, como biocatalisador, em modo batelada.

\section{MATERIAL E MÉTODOS}

\subsection{Material}

Os substratos utilizados nas reações de glicerólise enzimática foram o óleo de soja comercial (Soya, Brasil), glicerol (Vetec, 99,5\%) e hexano (Vetec, 99,5\%). Como catalisador foi utilizada a lipase comercial de origem microbiana Novozym 435, produzida a partir de Candida antarctica (imobilizada em resina acrílica macroporosa de troca iônica) pela Novozymes (Brasil/ Araucária-PR). Para a análise em GC foram utilizados diclorometano (Vetec, 99,5\%), derivatizante MSTFA (Nmethyl-N-trimethylsilyltrifluoroacetamide) e os padrões químicos monooleína, dioleína e trioleína adquirido da Sigma-Aldrich (Supelco, 99\% pureza).

\subsection{Aparato experimental}

A unidade experimental montada para o desenvolvimento do trabalho consistiu na utilização de um balão de fundo redondo de $50 \mathrm{~mL}$ que era inserido em um banho de ultrassom (UNIQUE), com frequência de $37 \mathrm{kHz}$ e potência de $132 \mathrm{~W}$, com controle de temperatura e potência do meio reacional. Neste sistema foi também inserido um agitador mecânico (IKA, modelo RW 20), com controlador digital. O procedimento experimental adotado na etapa de produção de MAGs e DAGs consistiu nos seguintes passos: primeiramente a enzima era colocada em estufa a $40{ }^{\circ} \mathrm{C}$ por 1 hora para secagem. Em seguida eram adicionados ao reator o óleo de canola/soja e o glicerol (em quantidades definidas no planejamento de experimentos, com volume total de $15 \mathrm{~mL}$ ), e a enzima em concentrações pré-estabelecidas. A relação mássica (óleo, glicerol e enzima) utilizada em cada reação foi determinada pelo planejamento experimental. Era acoplado ao ultrassom o agitador mecânico ao reator e este era fechado e a reação iniciada. Ao final do tempo da reação, todo o volume do reator era retirado e filtrado a vácuo com sucessivas lavagens com hexano. As amostras eram transferidas para frascos de amostragem e acondicionadas em estufa a vácuo $\left(65^{\circ} \mathrm{C}, 0,5\right.$ bar $)$ por $24 \mathrm{~h}$ a fim de eliminar o excesso de hexano.

\subsection{Determinação de MAG e DAG}

As análises das amostras foram realizadas em cromatógrafo gasoso (GC), - Shimadzu 2010, com injetor automático on-column e detector de ionização de chama (FID). Utilizou-se a coluna capilar DB-5 (5\% fenil, 95\% metilsiloxano) de $30 \mathrm{~m}$ x 0,25 mm x 0,1 $\mu \mathrm{m}$ (J e W Scientific, $\mathrm{n}^{\circ}$ de série: US7162944H). As condições de operação foram segundo a Norma $\mathrm{n}^{\circ} 14105$, do Comitê Europeu para Padronizações, sendo a programação de temperatura da coluna: $50{ }^{\circ} \mathrm{C}$ por 1 minuto, 


\section{9 a 22 de outubro de 2014 \\ Florianópolis/SC}

seguido pelo aumento de $15{ }^{\circ} \mathrm{C} / \mathrm{min}$ até $180{ }^{\circ} \mathrm{C}, 7{ }^{\circ} \mathrm{C} / \mathrm{min}$ até $230{ }^{\circ} \mathrm{C}$ e $10{ }^{\circ} \mathrm{C} / \mathrm{min}$ até $380{ }^{\circ} \mathrm{C}$, permanecendo por $8 \mathrm{~min}$. A temperatura do detector era de $380{ }^{\circ} \mathrm{C}$, pressão do gás de arraste (nitrogênio) de $80 \mathrm{kPa}$ e o volume injetado foi de $1 \mu \mathrm{L}$. Para a quantificação dos produtos MAG e DAG utilizou-se padrões externos monooleína e dioleína. As condições de análise das soluções de calibração e das amostras foram segundo a Norma $\mathrm{n}^{\circ} 14.105$ (2001) do Comitê Europeu para Padronizações, porém, sem a utilização de padrões internos conforme metodologia adaptada por Kruger (2010).

\section{RESULTADOS E DISCUSSÃO}

\subsection{Avaliação do efeito das variáveis de processo na produção de MAG E DAG}

Tomando como base estudos preliminares realizados por Fiametti et al. (2012) foi proposta a utilização do planejamento de experimentos através do modelo proposto por Placket \& Burman com 12 ensaios, com triplicata do ponto central, para avaliação dos efeitos das variáveis temperatura, razão molar, concentração de enzima, agitação e potência de ultrassom na produção enzimática de mono- e diacilgliceróis utilizando como catalisador a enzima Novozym 435, em meio livre de solvente orgânico, em banho de ultrassom. O tempo de reação foi fixado em 3 horas. A Tabela 1 apresenta a matriz do planejamento de experimento e as respostas em termos de rendimento em MAG, DAG e MAG+DAG utilizando óleo de soja.

Tabela 1 - Matriz do planejamento fatorial do tipo Plackett \& Burman (valores codificados e reais)

com resposta em termos de teor de monoacilgliceróis (MAG) e diacilgliceróis (DAG) $(\%, \mathrm{~m} / \mathrm{m})$

\begin{tabular}{ccccccccc}
\hline Ensaio & $\begin{array}{c}\text { Temp. } \\
\left({ }^{\circ} \mathrm{C}\right)\end{array}$ & $\begin{array}{c}\text { Razão* } \\
\text { Molar }\end{array}$ & $\begin{array}{c}\text { Conc. De } \\
\text { Enzima } \\
(\%, \mathrm{~m} / \mathrm{m})\end{array}$ & $\begin{array}{c}\text { Agitação } \\
(\mathrm{rpm})\end{array}$ & $\begin{array}{c}\text { Potência } \\
(\%)\end{array}$ & $\begin{array}{c}\text { MAG } \\
(\%, \mathrm{~m} / \mathrm{m})\end{array}$ & $\begin{array}{c}\text { DAG } \\
(\%, \mathrm{~m} / \mathrm{m})\end{array}$ & $\begin{array}{c}\text { MAG+DAG } \\
(\%, \mathrm{~m} / \mathrm{m})\end{array}$ \\
\hline 1 & $1(70)$ & $-1(0,8: 1)$ & $1(10)$ & $-1(0)$ & $-1(40)$ & 5,91 & 4,18 & 10,46 \\
2 & $1(70)$ & $1(3: 1)$ & $-1(2,5)$ & $1(600)$ & $-1(40)$ & 10,01 & 23,08 & 33,09 \\
3 & $-1(40)$ & $1(3: 1)$ & $1(10)$ & $-1(0)$ & $1(100)$ & 1,20 & 2,18 & 3,38 \\
4 & $1(70)$ & $-1(0,8: 1)$ & $1(10)$ & $1(600)$ & $-1(40)$ & 30,13 & 32,32 & 62,45 \\
5 & $1(70)$ & $1(3: 1)$ & $-1(2,5)$ & $1(600)$ & $1(100)$ & 8,73 & 11,71 & 20,44 \\
6 & $1(70)$ & $1(3: 1)$ & $1(10)$ & $-1(0)$ & $1(100)$ & 2,30 & 6,61 & 8,91 \\
7 & $-1(40)$ & $1(3: 1)$ & $1(10)$ & $1(600)$ & $-1(40)$ & 6,19 & 14,06 & 20,25 \\
8 & $-1(40)$ & $-1(0,8: 1)$ & $1(10)$ & $1(600)$ & $1(100)$ & 6,74 & 8,01 & 14,75 \\
9 & $-1(40)$ & $-1(0,8: 1)$ & $-1(2,5)$ & $1(600)$ & $1(100)$ & 2,78 & 2,33 & 5,01 \\
10 & $1(70)$ & $-1(0,8: 1)$ & $-1(2,5)$ & $-1(0)$ & $1(100)$ & 2,67 & 6,22 & 8,89 \\
11 & $-1(40)$ & $1(3: 1)$ & $-1(2,5)$ & $-1(0)$ & $-1(40)$ & 0,24 & 1,56 & 1,80 \\
12 & $-1(40)$ & $-1(0,8: 1)$ & $-1(2,5)$ & $-1(0)$ & $-1(40)$ & 0,18 & 0,47 & 0,75 \\
13 & $0(55)$ & $0(1,9: 1)$ & $0(6,25)$ & $0(300)$ & $0(70)$ & 7,7 & 14,74 & 22,44 \\
14 & $0(55)$ & $0(1,9: 1)$ & $0(6,25)$ & $0(300)$ & $0(70)$ & 9,9 & 12,17 & 22,16 \\
15 & $0(55)$ & $0(1,9: 1)$ & $0(6,25)$ & $0(300)$ & $0(70)$ & 7,04 & 14,75 & 21,79 \\
\hline
\end{tabular}

Na Tabela 1 pode-se observar que o ensaio que apresentou maior rendimento foi o $4(62,45 \%)$, este ensaio corresponde à temperatura mais elevada $\left(70^{\circ} \mathrm{C}\right)$, menor razão molar entre os substratos de 
$(0,8: 1)$, maior concentração de enzima $(10 \% \mathrm{~m} / \mathrm{m})$, maior agitação $(600 \mathrm{rpm})$ e menor potência de ultrassom (40\%), em 3 horas de reação. Os dados apresentados na Tabela 7 e seus resultados quanto ao rendimento total da reação (\% MAG+DAG) foram tratados estatisticamente e os principais efeitos das variáveis são apresentados na Figura 12, onde se pode observar que a temperatura e a agitação apresentaram um efeito positivo e significativo $(\mathrm{p}<0,05)$ sobre a conversão em MAG+DAG.

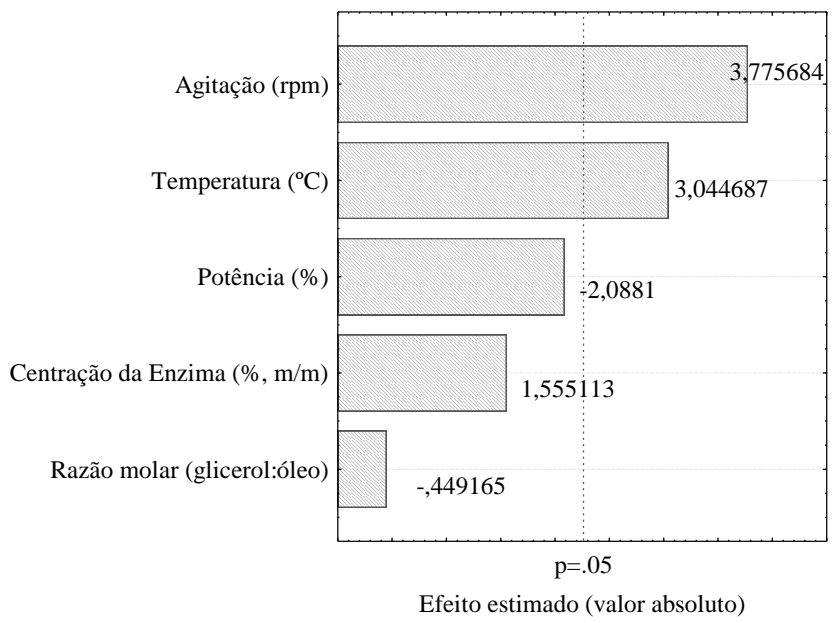

Figura 1 - Gráfico de Pareto para a produção enzimática de mono- e diacilgliceróis com a lipase Novozym 435 em sistema livre de solvente em banho de ultrassom com óleo de soja em função das variáveis independentes.

Os resultados em conversão em MAG e DAG ( 62\%) obtidos nesta primeira avaliação podem ser considerados relevantes, principalmente quando considerado o curto tempo de reação e a ausência de solventes e/ou surfactantes, que tornariam o processo mais oneroso e com maiores custos de produção e separação do produto final.

Com base nos resultados obtidos no planejamento Plackett \& Burman e na busca da maximização da produção de MAG e DAG, foram realizados planejamentos sequenciais, do tipo Delineamento Composto Central DCC $2^{3}$. Este planejamento também foi realizado com triplicata no ponto central a fim de calcular o erro experimental e em todos os experimentos o tempo de reação foi fixado em 3 horas. Nele manteve-se constante a concentração de enzima $(10 \% \mathrm{~m} / \mathrm{m})$ e razão molar (glicerol: óleo) $(0,8: 1)$, variando a temperatura, a potência do ultrassom e a agitação nas reações. A Tabela 2 apresenta a matriz do planejamento DCC $2^{3}$ com os valores codificados e reais, as respostas para a produção de MAG E DAG. O tratamento estatístico dos dados apresentados na Tabela 2 é apresentado na Figura 2. 
Tabela 2 - Matriz do planejamento de experimentos DCC $2^{3}$ (valores reais e codificados) com resposta em termos de teor de monoacilgliceróis (MAG) e diacilgliceróis (DAG) $(\%, \mathrm{~m} / \mathrm{m})$

\begin{tabular}{ccccccc}
\hline Ensaio & $\begin{array}{c}\text { Temperatura } \\
\left({ }^{\circ} \mathrm{C}\right)\end{array}$ & $\begin{array}{c}\text { Potência } \\
(\%)\end{array}$ & $\begin{array}{c}\text { Agitação } \\
(\mathrm{rpm})\end{array}$ & $\begin{array}{c}\text { MAG } \\
(\%, \mathrm{~m} / \mathrm{m})\end{array}$ & $\begin{array}{c}\text { DAG } \\
(\%, \mathrm{~m} / \mathrm{m})\end{array}$ & $\begin{array}{c}\text { MAG+DAG } \\
(\%, \mathrm{~m} / \mathrm{m})\end{array}$ \\
1 & $-1(60)$ & $-1(0)$ & $-1(600)$ & 16,64 & 25,39 & 42,03 \\
2 & $1(80)$ & $-1(0)$ & $-1(600)$ & 25,011 & 31,52 & 56,63 \\
3 & $-1(60)$ & $1(80)$ & $-1(600)$ & 23,49 & 29,01 & 52,51 \\
4 & $1(80)$ & $1(80)$ & $-1(600)$ & 24,73 & 31,83 & 56,57 \\
5 & $-1(60)$ & $-1(0)$ & $1(1200)$ & 23,64 & 25,75 & 49,39 \\
6 & $1(80)$ & $-1(0)$ & $1(1200)$ & 31,36 & 26,51 & 57,88 \\
7 & $-1(60)$ & $1(80)$ & $1(1200)$ & 25,01 & 31,87 & 56,88 \\
8 & $1(80)$ & $1(80)$ & $1(1200)$ & 28,10 & 29,37 & 57,47 \\
9 & $0(70)$ & $0(40)$ & $0(900)$ & 22,30 & 34,80 & 57,1 \\
10 & $0(70)$ & $0(40)$ & $0(900)$ & 20,85 & 41,25 & 62,12 \\
11 & $0(70)$ & $0(40)$ & $0(900)$ & 27,48 & 27,40 & 54,88 \\
\hline
\end{tabular}

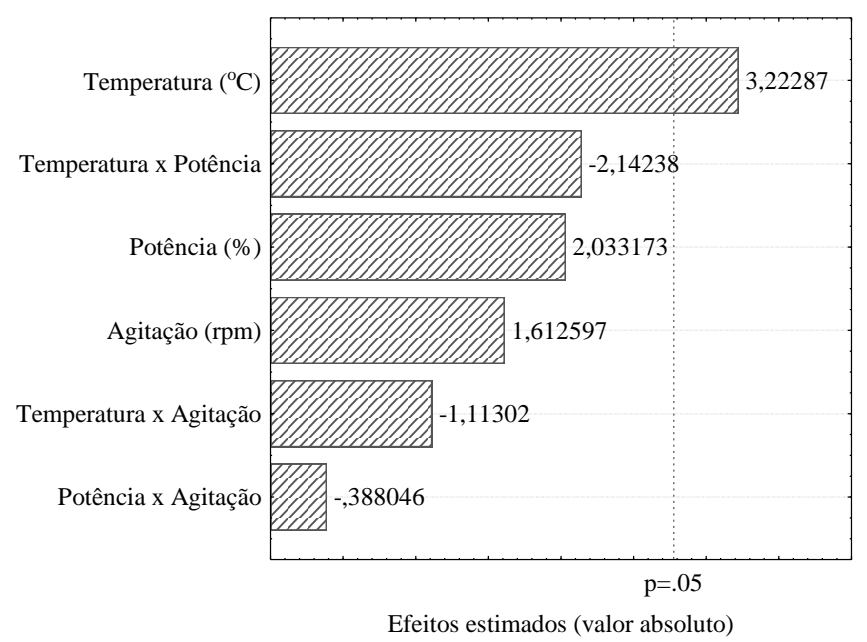

Figura 2 - Gráfico de Pareto para a produção enzimática de mono- e diacilgliceróis utilizando como substrato óleo de soja em função das variáveis independentes.

Em reações que envolvem o uso de glicerol, óleo e lipase imobilizada, um sistema trifásico é obtido, com uma fase oleosa hidrofóbica, uma fase hidrófila glicerol e uma fase sólida à qual a enzima é ligada (Pawongrat et al., 2007). Por isso, um obstáculo importante que tem de ser ultrapassado nestas reações é a baixa miscibilidade entre os substratos, óleo e glicerol (Feltes et al., 2012). A irradiação de ultrassom provoca a cavitação, fenômeno de formação e subsequente colapso de microbolhas de gás em um líquido. Quando bolhas da cavitação entram em colapso perto do limite de fase de dois líquidos imiscíveis, a onda de choque resultante pode proporcionar uma agitação 
muito eficiente, havendo uma mistura das camadas, aumentando as taxas de reação em reações heterogêneas (Yachmenev et al., 2004; Yoshimoto et al., 2005), evitando que o glicerol fiquei aderido à superfície da enzima.

\subsection{Cinética da produção de mono- e diacilgliceróis em sistema livre de solvente em banho de ultrassom}

A fim de avaliar o efeito da potência do ultrassom na síntese de MAG e DAG, foram mantidas fixas as condições: temperatura $70^{\circ} \mathrm{C}$, razão molar dos substratos de $0,8: 1$, concentração de enzima $10 \%(\mathrm{~m} / \mathrm{m})$ e agitação mecânica em $600 \mathrm{rpm}$, variando a potência do ultrassom em 0, 40, 80 e $100 \%$. O tempo definido para as realização das reações de glicerólise foi de 4 horas com amostragem destrutiva. As amostras foram retiradas nos tempos de 15, 30, 45, 60, 90, 120, 180 e 240 minutos

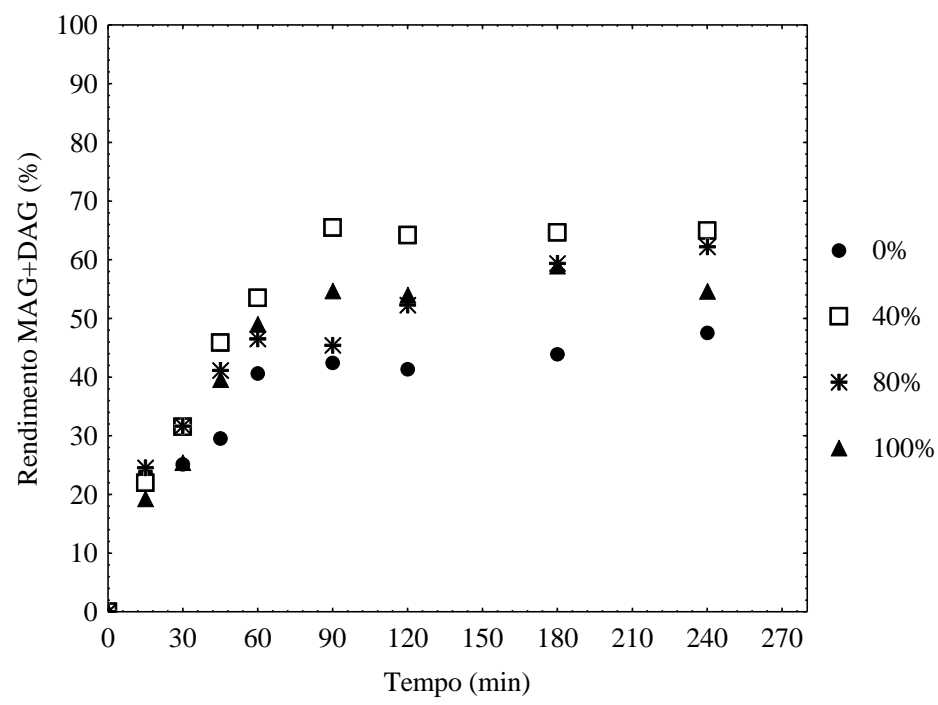

Figura 3 - Avaliação cinética da reação de glicerólise de óleo de soja em função da potência do ultrassom, em termos de conversão de MAG e DAG, na condição maximizada de produção (70

${ }^{\circ} \mathrm{C}$, razão molar dos substratos de $0,8: 1$, concentração de enzima $10 \%(\mathrm{~m} / \mathrm{m})$ e $600 \mathrm{rpm}$.

Na Figura 3 podem ser observadas as curvas quanto à avaliação cinética da reação de glicerólise de óleo de soja em função da potência do ultrassom em termos de conversão de MAG e DAG, na condição maximizada de produção. Pode-se observar que após 90 minutos de reação e 40\% de potência de ultrassom foram obtidos os maiores valores em conversão de MAG+DAG (65\%). Os altos rendimentos obtidos nas reações com irradiações, comparados aos valores obtidos quando se trabalhou sem irradiação de ultrassom $(0 \%)$, contribui para a teoria de que a irradiação de ultrassom pode ser considerada uma ferramenta alternativa para a melhoria dos processos químicos, físicos e biológicos, particularmente em sistemas de reação altamente viscosos e não miscíveis (Löning, Horst e Hoffmann, 2002).

\section{CONCLUSÃO}


De maneira geral, verificou-se que o uso do ultrassom nas reações de glicerólise enzimática dos óleos de soja contribuiu, em termos de eficiência do processo, diminuindo a resistência à transferência de massa no sistema glicerol/óleo/lipase imobilizada, para maximização da produção de MAGs e DAGs. O presente estudo contribuiu para o melhor entendimento da reação da glicerólise enzimática em sistema livre de solvente em banho de ultrassom, utilizando como substrato óleo de soja, que apresenta altos valores nutricionais e menores custos de mercado, utilizando como meio o ultrassom, uma tecnologia considerada limpa e viável economicamente, trazendo assim novas perspectivas para a produção de emulsificantes com alto valor de mercado.

\section{REFERÊNCIAS}

ANTUNES, O. A. C. Interfaces com a Indústria. Química Nova, v. 28, p. 64-75, 2005.

FELTES, M. M. C.; PITOL, L. O.; CORREIA, J. F. G.; GRIMALDI, R.; BLOCK, J. M.; NINOW, J.L. Incorporation of medium chain fatty acids into fish oil triglycerides by chemical and enzymatic interesterification. Grasas y Aceites, v. 60, n. 2, p. 168-176, 2009.

FELTES M. M. C., OLIVEIRA D., BLOCK J. M. \& NINOW J. L. The Production, Benefits, and Applications of Monoacylglycerols and Diacylglycerols of Nutritional Interest: review. Food and Bioprocess Technology, 2012.

FIAMETTI, K. G.; ROVANI, S.; OLIVEIRA, D.; CORAZZA, M. L.; TREICHEL, H.; OLIVEIRA, J. V. Kinetics of solvent-free lipase catalyzed production of monoacylglycerols from olive oil in Aerosol-OT surfactant. Industrial \& Engineering Chemistry Research, v. 48, n. 2, p. 708-712, 2012.

KRÜGER, R. Produção de mono e diacilgliceróis a partir da glicerólise enzimática de óleo de oliva. 2010. 131p. (Doutorado em Engenharia de Alimentos). Pós-Graduação em Engenharia de Alimentos, UFSC, Florianópolis.

LERIN, L. A. Produção enzimática de ascorbil palmitato em diferentes sistemas reacionais. 2010. (Doutorado em Bioquímica). Departamento de Bioquímica, UFRJ, Rio de Janeiro.

LÖNING, J.-M.; HORST, C.; HOFFMANN, U. Investigations on the energy conversion in sonochemical processes. Ultrason. Sonochem., 9, 169-179, 2002.

MARTINEZ, M.A.U.; DAVOLOS, M.R.; JAFELICCI M. J. O efeito do ultrassom em reações químicas. Química Nova, v. 23, p. 251-256, 2000.

PAWONGRAT, R., XU, X., \& H-KITTIKUN, A. Synthesis of monoacylglycerol rich in polyunsaturated fatty acids from tuna oil with immobilized lipase AK. Food Chem., 104, p. 251-258, 2007.

XU, X. Biocatalysis for lipid modifications. DUNFORD, N. T. e DUNFORD, H. B. (Ed.), 2004. 
YACHMENEV, V.G.; BLANCHARD, E.J.; LAMBERT, A.H. Use of ultrasonic energy for intensification of the bio-preparation of greige cotton, Ultrason., 42 87-91, 2004.

YOSHIMOTO M.; LI, C.; OGATA H.; TSUKUDA, N.; FUKUNAGA, K.; NAKAO, K. Effects of ultrasonic intensity and reactor scale on kinetics of enzymatic saccharification of various waste papers in continuously irradiated stirred tanks, Ultrason. Sonochem. 12, p. 373-384, 2005. 